\title{
Physiological and Dual Transcriptional Analysis of Microalga Graesiella emersonii-Amoeboaphelidium protococcarum Pathosystem Uncovers Conserved Defense Response and Robust Pathogenicity
}

\author{
Yi Ding ${ }^{1,2}$, Zhongjie Wang ${ }^{1,2}$, Yali Wang ${ }^{1,3}$, Yahong Geng ${ }^{1,2}$, Xiaobin Wen ${ }^{1,2, *}$ and Yeguang Li $^{1,2, * \mathbb{C}}$ \\ 1 CAS Key Laboratory of Plant Germplasm Enhancement and Specialty Agriculture, Wuhan Botanical Garden, \\ Chinese Academy of Sciences, Wuhan 430074, China; dingyi@wbgcas.cn (Y.D.); wzjihb@gamil.com (Z.W.); \\ wangyali20@mails.ucas.ac.cn (Y.W.); yahong@wbgcas.cn (Y.G.) \\ 2 Center of Economic Botany, Core Botanical Gardens, Chinese Academy of Sciences, Wuhan 430074, China \\ 3 University of Chinese Academy of Sciences, Beijing 100049, China \\ * Correspondence: xiaobin@wbgcas.cn (X.W.); yeguang@wbgcas.cn (Y.L.)
}

check for updates

Citation: Ding, Y.; Wang, Z.; Wang, Y.; Geng, Y.; Wen, X.; Li, Y.

Physiological and Dual

Transcriptional Analysis of Microalga

Graesiella emersonii-Amoeboaphelidium protococcarum Pathosystem Uncovers Conserved Defense Response and Robust Pathogenicity. Int. J. Mol. Sci. 2021, 22, 12847. https://doi.org/ $10.3390 /$ ijms222312847

Academic Editor: Fucheng Lin

Received: 28 October 2021

Accepted: 24 November 2021

Published: 27 November 2021

Publisher's Note: MDPI stays neutral with regard to jurisdictional claims in published maps and institutional affiliations.

Copyright: (c) 2021 by the authors. Licensee MDPI, Basel, Switzerland. This article is an open access article distributed under the terms and conditions of the Creative Commons Attribution (CC BY) license (https:// creativecommons.org/licenses/by/ $4.0 /)$.
Abstract: The underlying mechanisms of microalgal host-pathogen interactions remain largely unknown. In this study, we applied physiological and simultaneous dual transcriptomic analysis to characterize the microalga Graesiella emersonii-Amoeboaphelidium protococcarum interaction. Three infection stages were determined according to infection rate and physiological features. Dual RNA-seq results showed that the genes expression of $G$. emersonii and A. protococcarum were strongly dynamically regulated during the infection. For microalgal hosts, similar to plant defense response, the expression of defense genes involved in the pattern recognition receptors, large heat shock proteins, and reactive oxygen scavenging enzymes (glutathione, ferritin, and catalase) were significantly upregulated during infection. However, some genes encoding resistance proteins (R proteins) with a leucine-rich repeat domain exhibited no significant changes during infection. For endoparasite A. protococcarum, genes for carbohydrate-active enzymes, pathogen-host interactions, and putative effectors were significantly upregulated during infection. Furthermore, the genes in cluster II were significantly enriched in pathways associated with the modulation of vacuole transport, including endocytosis, phagosome, ubiquitin-mediated proteolysis, and SNARE interactions in vesicular transport pathways. These results suggest that $G$. emersonii has a conserved defense system against pathogen and that endoparasite $A$. protococcarum possesses a robust pathogenicity to infect the host. Our study characterizes the first transcriptomic profile of microalgae-endoparasite interaction, providing a new promising basis for complete understanding of the algal host defense strategies and parasite pathogenicity.

Keywords: oleaginous microalgae; Amoeboaphelidium protococcarum; dual RNA-seq; host defense response; pathogenicity

\section{Introduction}

Oleaginous microalgae have been suggested as a promising feedstock for biodiesel production [1]. In recent years, oleaginous microalgae cultivation, combined with wastewater treatment and $\mathrm{CO}_{2}$ mitigation, has been suggested as an environmentally sustainable process with the production of high value-added bioproducts [2,3]. However, the mass cultivation of microalgae is susceptible to microbial contamination (especially parasitic fungi), which can lead to algal culture crashes [4]. In natural ecosystems, algal parasites are a key driving factor in phytoplankton seasonal successions [5]. In the mass cultivation of microalgae, these parasites can cause microalgal population crashes and trigger further damage to their valuable products [6]. Knowledge about the isolation, identification, and 
progression of algal parasites is currently growing [7]. However, the underlying mechanisms of algal infection by fungal parasites remain largely unknown due to the lack of stable pathosystems for laboratory investigations [8,9].

The oleaginous microalga Graesiella emersonii is an industrial strain for lipid production [10], but it is frequently infected by endoparasite Amoeboaphelidium protococcarum belonging to the class Aphelidea. The infection progress starts with an amoeboid zoospore attached to an algal cell, followed by the formation of a cyst with a penetration tube [11]. Then, the propagule of A. protococcarum is penetrated into the host cell, thus triggering its intracellular development to gradually phagocytize the contents of the algal cell [4]. A. protococcarum exhibited high host specificity on G. emersonii and Scenedesmus dimorphus [7]. In a previous study, we created a stable and laboratory G. emersonii and A. protococcarum pathosystem. Hence, this pathosystem can enable the investigation of microalgalfungal parasite interactions.

In addition, recent studies have characterized the molecular defensive mechanisms of macroalgae using the algal pathosystem. For instance, studies on the brown alga Ectocarpus siliculosus and the oomycete Eurychasma dicksonii have demonstrated that E. siliculosus responds to pathogen infection by strengthening the cell wall and accumulating reactive oxygen species (ROS) and putative halogen metabolism [12]. In 2019, Im et al. identified the genes involved in the interaction of the red alga Pyropia tenera and three pathogens using microarray analysis and histochemical methods [13]. Tang et al. identified the genes associated with the defense response of the red alga Pyropia yezoensis against the necrotrophic pathogen Pythium porphyrae using transcriptomic analysis [14]. However, microalgal responses and defense reactions to pathogen infection at the molecular level have never been investigated.

In this study, physiological and simultaneous dual transcriptomic analyses were conducted to characterize the G. emersonii-A. protococcarum interaction for the first time. We aimed to understand the host algal defense strategies and algal parasite pathogenicity during the interaction between G. emersonii and A. protococcarum. These results may provide new insights into microalgal host responses to endoparasites or the identification of pathogen-host interaction genes.

\section{Results}

\subsection{Analysis of the Symptom and Features Associated with Infection by A. protococcarum}

Microscopic observation of the infection progress revealed that $A$. protococcarum was an endoparasite that can occupy the microalgal host cell and replace the host cytoplasm (Figure 1a). In the infection test, the infection rates of G. emersonii by A. protococcarum gradually increased over time (Figure 1b). In contrast, healthy and uninfected G. emersonii cells did not exhibit any symptoms during cultivation. Infection stages were determined based on the infection rate. Briefly, healthy and uninfected G. emersonii cells were used as controls (GA). At 3 dpi, the infection rate of G. emersonii reached approximately $5 \%$ and was defined as early stage infection (ES). At $4 \mathrm{dpi}$, the infection rate reached approximately $50 \%$ and was defined as medium stage (MS). At $5 \mathrm{dpi}$, the infection rate reached approximately $90 \%$ in G. emersonii cells and was defined as late stage (LS). Samples of GA, ES, MS, and LS were collected and stored in liquid nitrogen for subsequent dual transcriptomic analysis.

The ROS content and Fv/Fm values of G. emersonii cells during infection were measured, and the results are shown in Figure 1c,d. With the increase in culture time, ROS levels gradually increased. At 1, 2, and $3 \mathrm{dpi}$, the values of $\mathrm{Fv} / \mathrm{Fm}$ were not significantly decreased compared with those of the control. At 5 dpi, Fv/Fm values decreased significantly. 
a
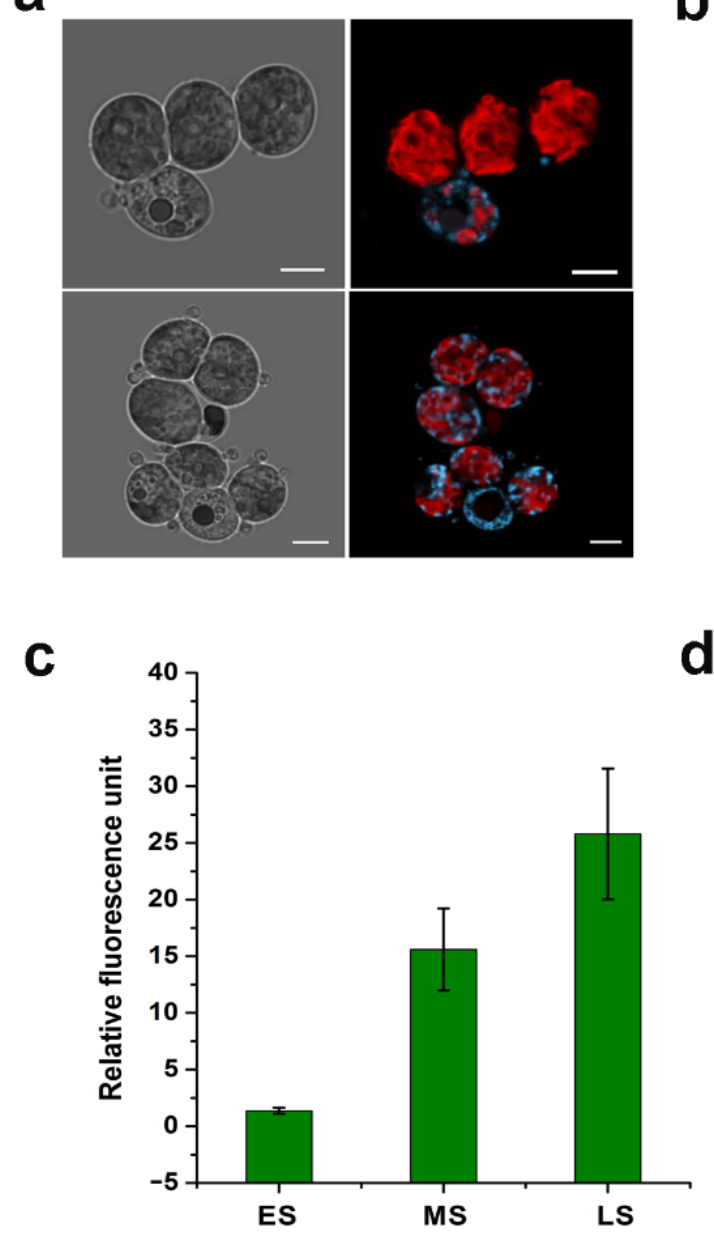

b

d
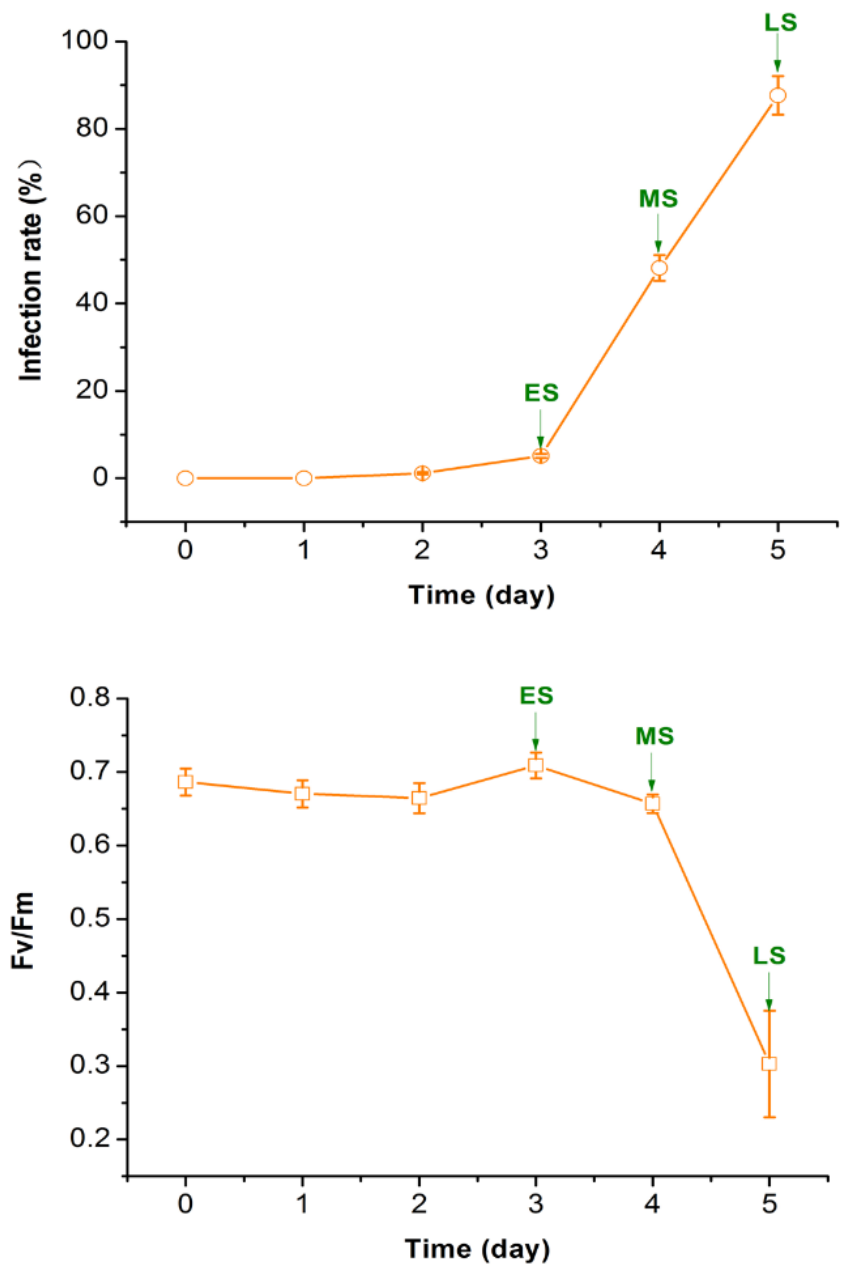

Figure 1. Symptoms and physiological features of Graesiella emersonii due to Amoeboaphelidium protococcarum infection. (a) Typical symptom associated with infection by $A$. protococcarum under fluorescence microscope, scale bars $=5 \mu \mathrm{m}$; (b) infection rate; (c) the relative ROS levels; (d) photosynthetic activity (Fv/Fm).

\subsection{A Global View of Transcriptomic Analysis of Microalga and Endoparasite during Their Interaction}

To characterize the gene expression profiles in the host microalga G. emersonii and endoparasite $A$. protococcarum during infection, we performed a dual RNA-seq analysis at different infection stages. A total of $102 \mathrm{~Gb}$ of clean bases were generated from 12 samples, and sequencing data quality is summarized in Table S1. For the microalgal host, 1843, 1750, and 2164 differentially expressed genes (DEGs) were upregulated, whereas 1818, 1168, and 1139 DEGs were downregulated in ES, MS, and LS compared with GA, respectively (Figure 2a). We found that more genes were upregulated, while fewer genes were downregulated in MS. As the stages of infection progressed, the ratio of upregulated genes to the whole DEGs increased from $50.3 \%$ at ES to $65.5 \%$ at LS. Figure 2 a illustrates that only 608 DEGs overlapped with ES, MS, and LS, suggesting that the microalgal response due to infection is different at the molecular level in three stages. For fungal parasites, a total of 11,352 and 17,819 upregulated DEGs were detected in MS and LS compared with the ES group (Figure $2 \mathrm{~b}$ ). These genes may play a key role in the progression of infection. In addition, Pearson correlation coefficients of the RNA-Seq 12 samples are displayed in Figure 2c. 
a

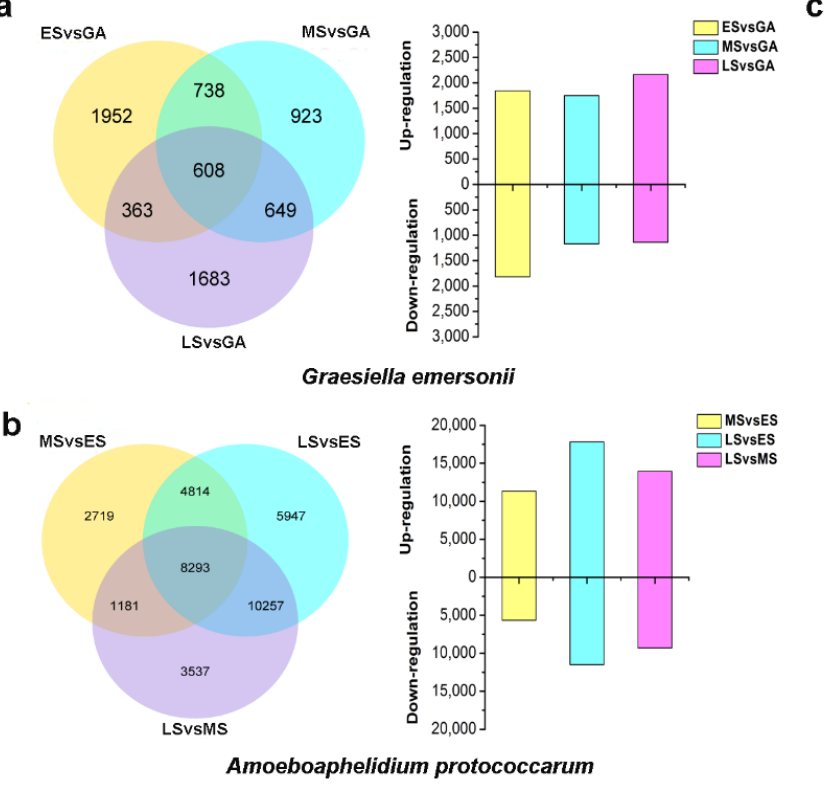

C

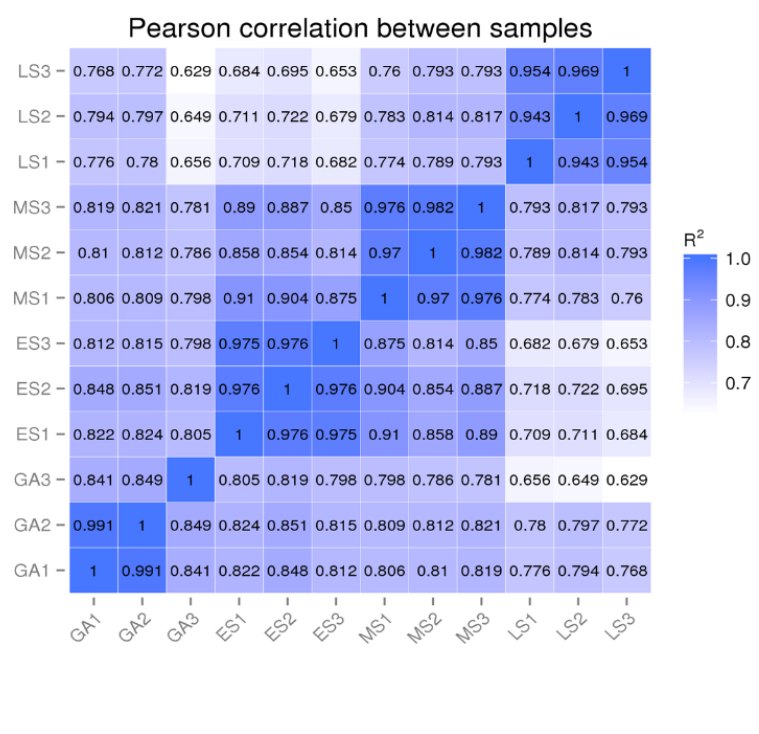

Figure 2. Global view of differentially expressed genes in G. emersonii and A. protococcarum during infection. (a) G. emersonii; (b) A. protococcarum; (c) Pearson correlation coefficients of all 12 samples.

\subsection{Gene Expression Profile of G. emersonii during Infection}

To further investigate the gene expression profile of G. emersonii during infection, the gene expression of the host G. emersonii was clustered using the short time-series expression miner (STEM). A total of 23,248 genes were divided into 50 profiles, but only 22 highly significant expression profiles were chosen for further functional analysis $(p<0.001)$ (Figure S1). The 22 profiles were then grouped into seven major clusters based on their expression patterns (Figure 3a). Compared with the GA, cluster I comprised profiles 27,28 , and 41 , where the genes were generally upregulated in both ES and MS stages and were not affected in LS stages. Genes in cluster II, combining profiles 18, 7, and 5, were downregulated in the ES stage but upregulated in both the MS and LS stages. Cluster III (profiles 21, 30, 29, and 20) included genes whose expression was generally upregulated but fluctuated. Genes in cluster IV showed an overall downregulation. The genes in cluster $\mathrm{V}$ were downregulated but experienced some variability. The majority of genes in cluster VI were upregulated or were stable in the ES and MS stages and downregulated in the LS stage. The genes in cluster VII were generally upregulated in ES and stable in the MS and LS stages.

To elucidate the biological function in each cluster, GO term enrichment and KEGG pathway analyses were conducted for significant enrichment. The top six GO terms for each cluster are shown in Figure 3b. Cluster I, which peaked in expression at the MS stage, was significantly enriched for genes involved in hydrolase activity. The DEGs in cluster II were enriched with many ligase activity-related GO terms, cluster VI was enriched with numerous oxidoreductase-related terms, while cluster IV showed no significant results.

KEGG pathway analysis showed that seven clusters mapped significantly with 20 pathways (Figure 3c). Notably, cluster I was enriched in the ubiquitin-mediated proteolysis pathway, endocytosis, fatty acid biosynthesis, and fatty acid metabolism. Multiple clusters were enriched with endocytosis, proteasome, protein processing in the endoplasmic reticulum, and spliceosome.

\subsection{Ubiquitin Mediated Proteolysis and Endocytosis in Response to Infection}

Given the above-mentioned enriched pathways in the microalga G. emersonii during infection, we focused on the pathways of ubiquitin-mediated proteolysis and endocytosis. In the transcriptome data, the expression of genes encoding enzyme E1 (gene-Cem18475, gene-Cem15045) was upregulated by 2.2- and 1.1- $\log _{2}$ fold changes, respectively (Figure 4a). 
The expression of genes encoding E2 (gene-Cem18885, gene-Cem21008, gene-Cem11452, and gene-Cem01030) also experienced a significant increase. In contrast, the expression of several genes encoding E3 was downregulated. Moreover, the genes encoding other key enzymes, such as heat shock proteins (HSPs), which are also involved in the endocytosis pathway, were induced.

\subsection{Potential Pathogen Receptors and Putative R Proteins in G. emersonii}

In G. emersonii, genes that contained typical pattern-recognition receptor (PRR) functional domains were searched using Pfam annotation. Epidermal growth factor (EGF)containing, lectin-containing genes, leucine-rich repeat (LRR)-containing genes, and LysMcontaining genes with upregulated expression between one or more infection stages are summarized in Figure 4b. Among these genes, EGF (gene-Cem19681), lectin-containing genes (gene-Cem18265, gene-Cem18266), and LRR-containing genes (gene-Cem02208, gene-Cem04600, gene-Cem11344, gene-Cem14526, gene-Cem14758, gene-Cem16818) were predicted as putative transmembrane proteins using TMHMM v. 2.0. At the same time, LRR-containing genes (gene-Cem08139, gene-Cem08774, gene-Cem12703, gene-Cem13414, and gene-Cem16818) significantly increased in both ES and MS during infection. The LRR-containing gene (gene-Cem02208) was upregulated in ES by 5.6-fold. Moreover, four genes encoding putative R proteins with an LRR domain (gene-Cem07770, gene-Cem16366, gene-Cem22200, and Novel00913) were found, but none were significantly upregulated at the three infection stages. No genes encoding the TIR or NBS domain were detected in the G. emersonii transcripts.

a

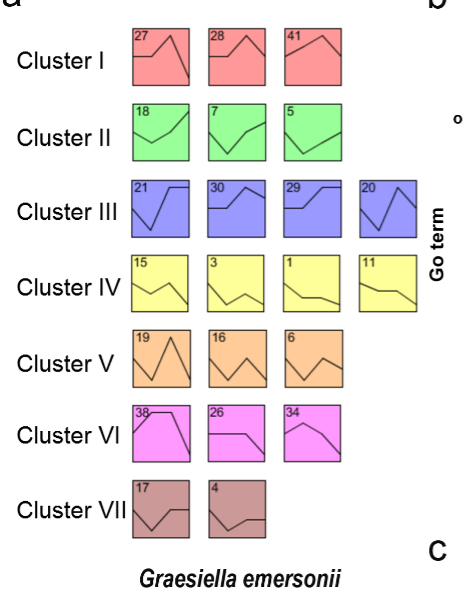

b
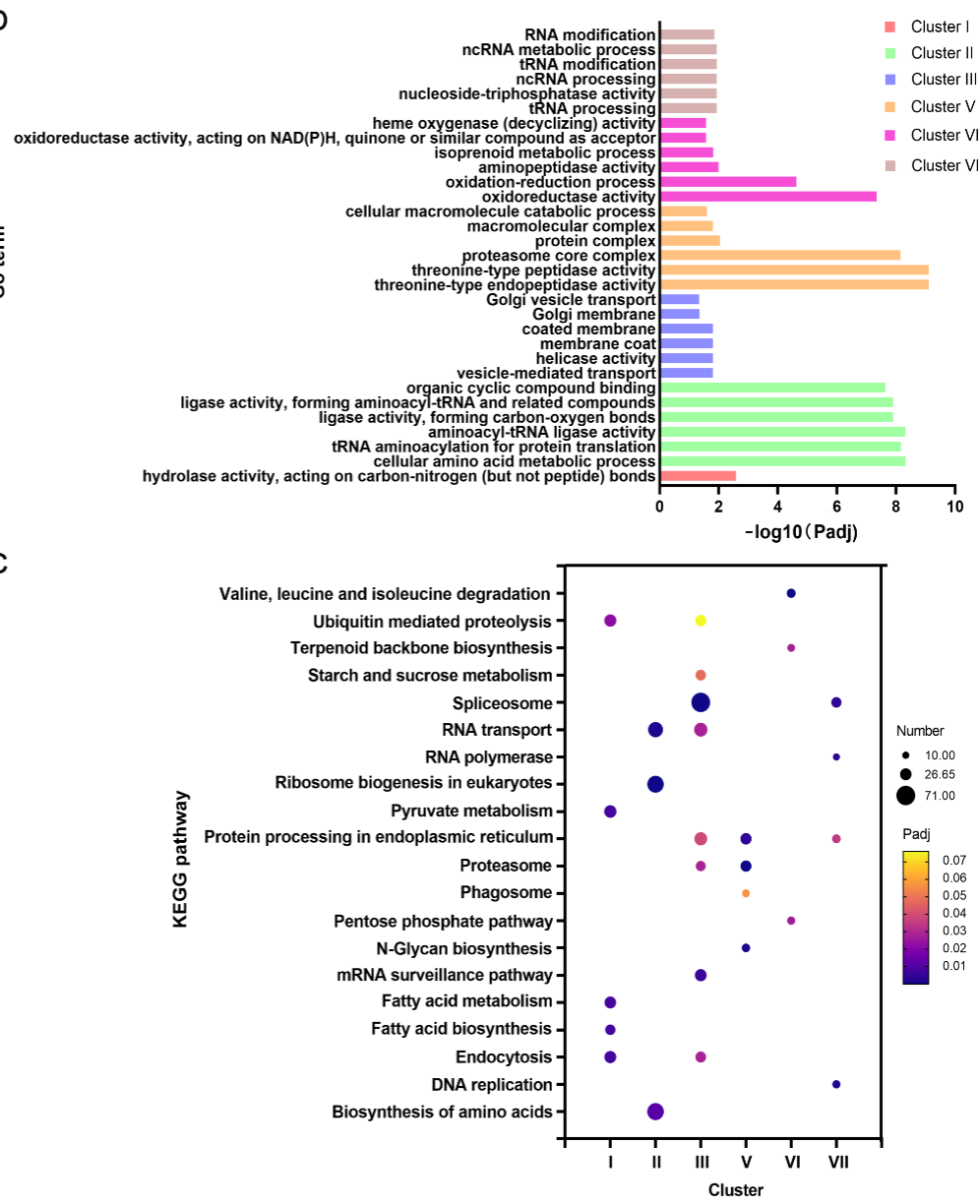

Figure 3. STEM trend and functional enrichment analysis of microalga G. emersonii during infection. (a) Seven clusters by STEM analysis; (b) GO terms enrichment analysis; (c) KEGG pathway enrichment analysis. 
a

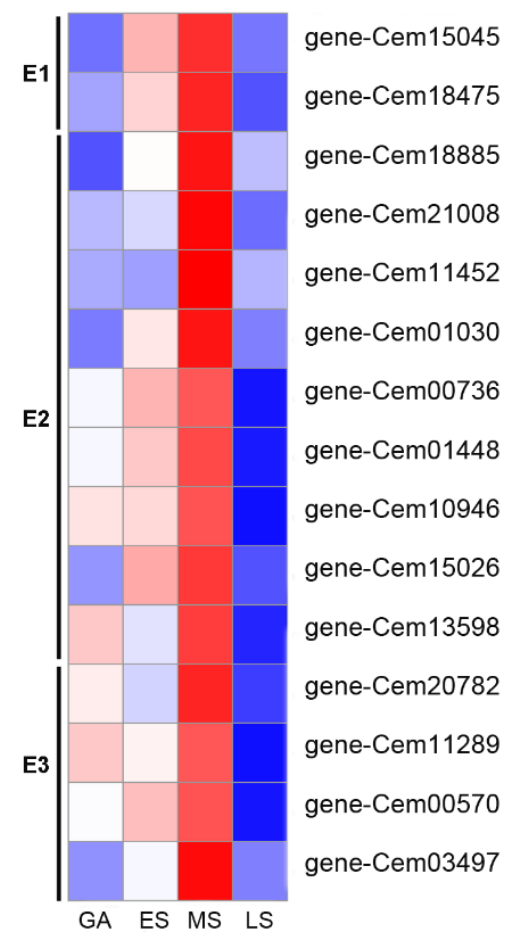

C

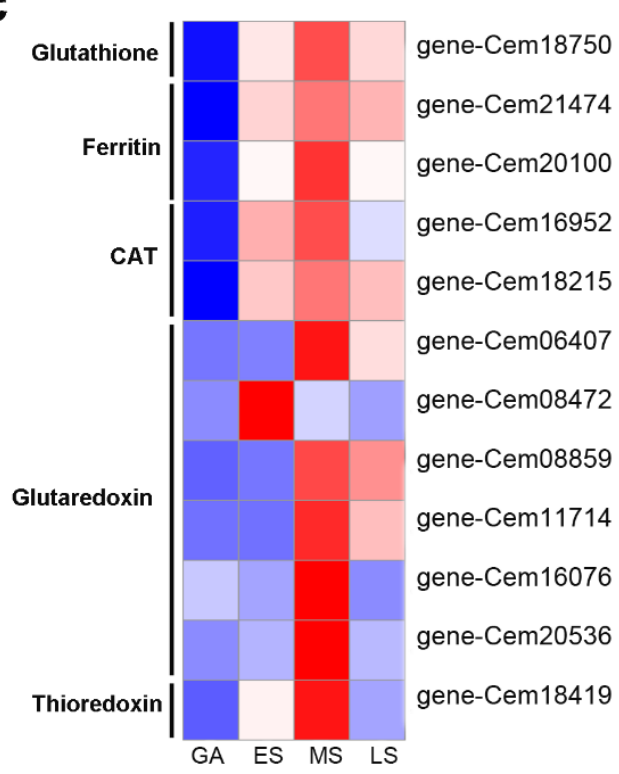

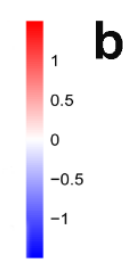

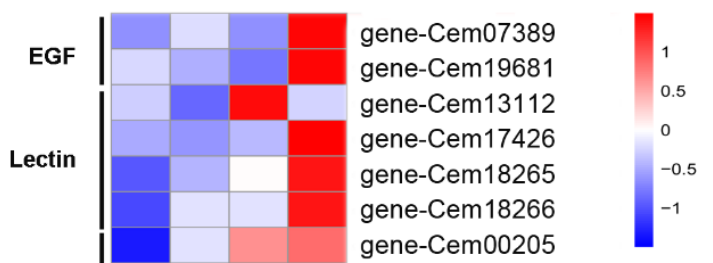

LRR

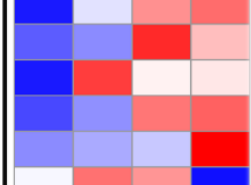

gene-Cem02208

gene-Cem04600

gene-Cem06303

gene-Cem08139

gene-Cem08774

gene-Cem09108

gene-Cem11344

gene-Cem12703

gene-Cem12704

gene-Cem13414

gene-Cem14526

gene-Cem14758

gene-Cem16818

gene-Cem17338

gene-Cem12702

gene-Cem13890

gene-Cem14313

d

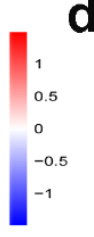

gene-Cem00504

gene-Cem11343

gene-Cem07833

gene-Cem06729

gene-Cem06727

gene-Cem07830

gene-Cem07831

gene-Cem01836

gene-Cem16785

gene-Cem15267

gene-Cem18798

gene-Cem18799

Figure 4. Heatmap of defense-related genes in G. emersonii during infection. (a) Ubiquitin-proteasome system (UPS)related genes; (b) typical pattern-recognition receptor (PRR)-related genes; (c) ROS-related genes; (d) heat shock proteins (HSPs)-related genes.

\subsection{ROS-Related Genes in Response to Infection}

The generation of superfluous ROS due to pathogens may cause oxidative stress and cell damage. G. emersonii can protect themselves by various ROS-scavenging enzymes. Our results demonstrate that the expression of genes encoding glutathione (gene-Cem18750), ferritin (gene-Cem21474), and CAT (gene-Cem18215) were upregulated at ES and maintained at high levels in MS and LS (Figure 4c). The genes encoding glutaredoxin (gene-Cem06407, gene-Cem11714) were not significantly modified at ES, but were upregulated in MS and LS. Notably, the gene encoding for ferritin (gene-Cem21474) was upregulated by $7.9 \log _{2}$ fold change at ES, $8.4 \log _{2}$ fold change at MS, and $8.1 \log _{2}$ fold change at LS. The expression of genes encoding glutathione (gene-Cem18750) was also dramatically increased by 
$5.3 \log _{2}$ fold change at ES, $6.6 \log _{2}$ fold change at MS, and $5.4 \log _{2}$ fold change at MS. These transcriptional results confirmed that the upregulation of genes encoding ROS-scavenging enzymes is one of the defense strategies of G. emersonii during infection.

\subsection{Some Heat Shock Proteins (HSPs) and Transcription Factors (TFs) Involved during Infection}

The results of this study show that 12 genes encoding heat shock proteins were differentially regulated during infection (Figure $4 \mathrm{~d}$ ). Compared with the uninfected control group, five HSP genes (four HSP90 and one HSP70) were upregulated in one or more infection stages.

RNA-seq analysis revealed 774 differentially expressed TFs in G. emersonii (see Table S2). SET, SNF2, GNAT, MYB, AP2-EREBP, and TRAF were the top six differentially expressed TFs. Among them, the identified five TFs that were upregulated during the infection included two SNF2, one PLATZ, one FHA, and one orphan (Table 1).

Table 1. Transcriptional factors of G. emersonii that were continuously upregulated because of infection.

\begin{tabular}{cccccc}
\hline No. & Gene ID & Types & Log2FC(ESvsGA) & Log2FC(MSvsGA) & Log2FC(LSvsGA) \\
\hline 1 & gene-Cem00854 & PLATZ & 1.65 & 3.61 & 3.06 \\
2 & gene-Cem04263 & SNF2 & 1.53 & 2.31 & 1.65 \\
3 & gene-Cem15036 & FHA & 2.91 & 2.45 & 1.58 \\
4 & gene-Cem16321 & SNF2 & 2.08 & 3.57 & 2.51 \\
5 & gene-Cem19050 & Orphans & 1.41 & 2.68 & 3.18 \\
\hline
\end{tabular}

$\log _{2} \mathrm{FC}=\log _{2}$ fold change.

\subsection{Gene Expression Profile of A. protococcarum during Infection}

STEM analysis was carried out to elucidate the gene expression profile of $A$. protococcarum during infection. In total, 16 profiles were generated, but only 7 highly significant expression profiles were chosen for further functional analysis $(p<0.001)$ (see Figure S2). The seven profiles were subsequently grouped into three clusters based on their expression patterns (Figure 5a). Cluster I comprised profile 7, 2, 3, and 0, where the genes were generally downregulated compared with those in ES. Genes in cluster II, which combined profiles 13 and 6, were upregulated. Genes in cluster III were upregulated and then downregulated.

GO term enrichment and KEGG pathway analyses were also conducted for the three clusters of A. protococcarum. The top $10 \mathrm{GO}$ terms for each cluster are shown in Figure $5 \mathrm{~b}$. The genes involved in macromolecular complex, intracellular organelle, and organic substance biosynthetic processes that tended toward downregulation were related to cluster I. The genes associated with the regulation of GTPase activity and response to host immune that showed an upregulated trend were in cluster II. The genes in cluster III were enriched in the establishment of localization in cells, cellular localization, and vesiclemediated transport GO terms. These GO terms were both necessary and significant to A. protococcarum infection.

KEGG pathway analysis demonstrated that the three clusters in A. protococcarum were mapped with 25 pathways (Figure 5c). Interestingly, cluster II, featuring an upregulated trend over time, was enriched in glycosylphosphatidylinositol (GPI)-anchor biosynthesis, endocytosis, phagosome, ubiquitin-mediated proteolysis, SNARE interactions in vesicular transport, MAPK signaling pathway, and amino sugar and nucleotide sugar metabolism.

\subsection{Expression of CAZymes and Pathogen-Host Interaction Genes in A. protococcarum during Infection}

To assess the potential of $A$. protococcarum to depolymerize the cell walls of microalgal hosts, CAZymes-containing genes were searched using the dbCAN2 meta server (HMMER). There were 69 putative genes encoding CAZymes differentially expressed and identified in cluster II of A. protococcarum, which exhibited an upregulated temporal trend (see Table S3). CAZymes were classified into glycoside hydrolases (GHs), glycosyltransferases (GTs), and auxiliary activities (AAs) super families. Among them, two genes (Cluster-8366.7755 
and Cluster-8366.85495) encoding $\alpha$-glucosidase were upregulated by 6.2- and 10.7-log2 fold changes in LS, respectively. Two genes (Cluster-8366.11810 and Cluster-8366.64947) encoding trehalase and one gene (Cluster-8366.6141) encoding cellulase experienced a dramatic increase as well. The expression of genes encoding mannosyltransferase, galactosyltransferase, and glucosyltransferase was significantly upregulated during infection. It should be emphasized that several CAZymes genes associated with chitin binding and chitin synthase (AA15 and GT2) were also significantly upregulated, which might play a role in the chitin synthesis of A. protococcarum.
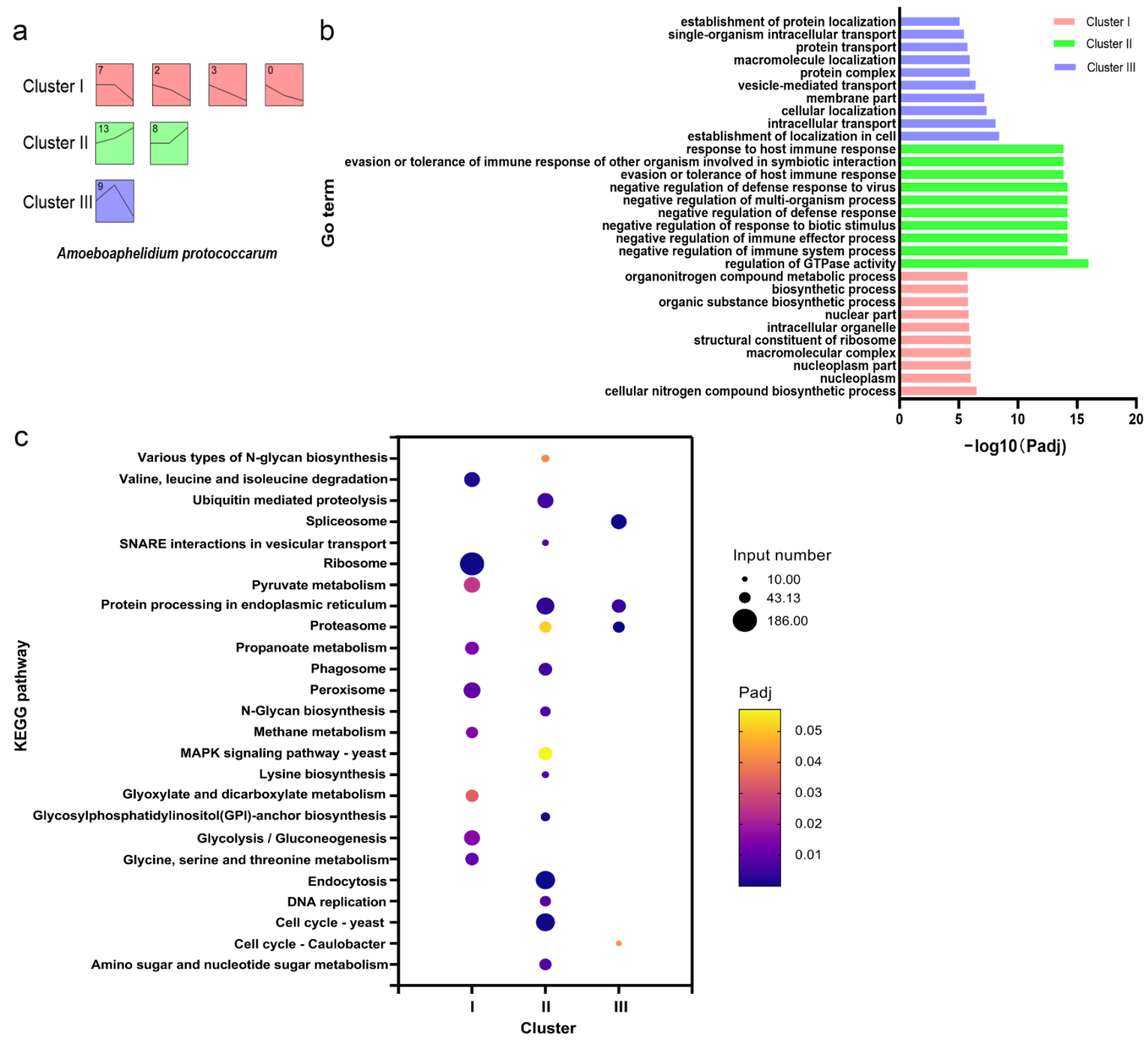

Figure 5. STEM trend and functional enrichment analysis of endoparasite A. protococcarum during infection. (a) Three clusters by STEM analysis; (b) GO terms enrichment analysis; (c) KEGG pathway enrichment analysis.

To predict key genes involved in infection, the pathogenicity genes of A. protococcarum were predicted using BLASTp against the Pathogen-Host Interaction database (PHI-base). In total, 1269 genes associated with key virulence and pathogenicity, accounting for $5.3 \%$ of the total predicted genes, were identified (see Table S4). Most genes $(1231.97 \%$ ) were expressed in the transcriptome of A. protococcarum during infection. Among them, 72 genes associated with increased virulence (Table S5) and 15 genes associated with effectors 
were significantly expressed at all infection stages (Table 2). Furthermore, 11 putative secretory protein-encoding genes were identified in pathogen-host interaction genes to seek for candidate virulence and effectors in A. protococcarum. However, only a candidate hypervirulence gene (Cluster-8366.11022) was upregulated by 2.1-, 5.9-, and 3.5-log2 fold change during infection. Two candidate effectors (Cluster-9881.0, Cluster-8366.6798) were upregulated by 2.4- and 2.5- $\log 2$ fold changes, during infection.

Table 2. Fifteen genes associated with effector in A. protococcarum were significantly upregulated in all the infection stages.

\begin{tabular}{|c|c|c|c|c|c|c|c|c|}
\hline No. & Gene ID & ProteinID & PHI ID & Gene Name & Phenotype & $\log 2 \mathrm{FC}(\mathrm{MS}$ vs. ES) & $\log 2 \mathrm{FC}(\mathrm{LS}$ vs. ES) & $\log 2 \mathrm{FC}(\mathrm{LS}$ vs. MS) \\
\hline 1 & $\begin{array}{c}\text { Cluster- } \\
8366.85734\end{array}$ & A0А0H3НVK0 & PHI:5335 & $c l p V-5$ & Effector & 1.729 & 3.8033 & 1.8221 \\
\hline 2 & $\begin{array}{c}\text { Cluster- } \\
8366.8318\end{array}$ & Q8RP09 & PHI:981 & hopI1 & Effector & 2.0296 & 6.6465 & 4.3176 \\
\hline 3 & $\begin{array}{c}\text { Cluster- } \\
8366.82670\end{array}$ & Q79LY0 & $\begin{array}{c}\text { PHI:992/ } \\
\text { PHI:7237/ } \\
\text { PHI:7265 }\end{array}$ & $\begin{array}{c}\text { hopPtoD2/ } \\
\text { hopAO1/ } \\
\text { HopPtoD2 }\end{array}$ & Effector & 1.6504 & 4.9986 & 3.0935 \\
\hline 4 & $\begin{array}{c}\text { Cluster- } \\
8366.80929\end{array}$ & P17778 & $\begin{array}{c}\text { PHI:6101/ } \\
\text { PHI:6824/ } \\
\text { PHI:6830 }\end{array}$ & YopM & Effector & 6.0254 & 11.636 & 3.4623 \\
\hline 5 & $\begin{array}{c}\text { Cluster- } \\
8366.7571\end{array}$ & C5BD30 & PHI:6294 & EseM & Effector & 2.5059 & 7.5318 & 4.6832 \\
\hline 6 & $\begin{array}{c}\text { Cluster- } \\
8366.72579\end{array}$ & Q8PC98 & PHI:7945 & pip & Effector & 4.4021 & 9.7346 & 2.9476 \\
\hline 7 & $\begin{array}{c}\text { Cluster- } \\
8366.6633\end{array}$ & Q8PC98 & PHI:7945 & pip & Effector & 3.5808 & 10.373 & 4.6194 \\
\hline 8 & $\begin{array}{c}\text { Cluster- } \\
8366.43382\end{array}$ & Q8XTK9 & PHI:5119 & RSp0099 & Effector & 1.7086 & 4.8665 & 2.9109 \\
\hline 9 & $\begin{array}{l}\text { Cluster- } \\
8366.14241\end{array}$ & P17778 & $\begin{array}{c}\text { PHI:6101/ } \\
\text { PHI:6824/ } \\
\text { PHI:6830 }\end{array}$ & YopM & Effector & 1.6503 & 5.6023 & 3.6416 \\
\hline 10 & $\begin{array}{c}\text { Cluster- } \\
8366.11799\end{array}$ & Q8PI08 & PHI:2703 & xac3090 & Effector & 1.5342 & 5.0159 & 3.2262 \\
\hline 11 & $\begin{array}{l}\text { Cluster- } \\
8366.11711\end{array}$ & Q8XZN9 & PHI:5173 & RSc1356 & Effector & 2.971 & 6.0476 & 2.8247 \\
\hline 12 & $\begin{array}{c}\text { Cluster- } \\
8366.11710\end{array}$ & Q8XZN9 & PHI:5173 & RSc1356 & Effector & 2.3996 & 5.6692 & 3.0096 \\
\hline 13 & $\begin{array}{c}\text { Cluster- } \\
8366.11150\end{array}$ & Q8XZN9 & PHI:5173 & RSc1356 & Effector & 3.3433 & 7.5956 & 3.9299 \\
\hline 14 & $\begin{array}{c}\text { Cluster- } \\
8366.11149\end{array}$ & Q8XZN9 & PHI:5173 & $R S c 1356$ & Effector & 3.8592 & 11.787 & 3.2499 \\
\hline 15 & $\begin{array}{c}\text { Cluster- } \\
8366.11148\end{array}$ & Q8XZN9 & PHI:5173 & $R S c 1356$ & Effector & 3.1417 & 7.4909 & 4.0076 \\
\hline
\end{tabular}

\section{Discussion}

Mass cultivation of microalgae for biodiesel production is susceptible to epidemics caused by various pathogens [15]. Thus, it is essential to investigate microalgae-pathogen interactions. Dual RNA sequencing opens new prospects for investigating the underlying molecular mechanisms of microalgal host-pathogen interplay. However, dual transcriptional profiling of microalgae-pathogens is lacking. In this study, the experimental and stable pathosystem between oleaginous microalga G. emersonii and its endoparasite A. protococcarum was established. Using simultaneous dual transcriptomics, we dissected G. emersonii genes related to basic defense and A. protococcarum genes related to pathogenesis involved in the microalga-pathogen interaction. The basic interaction of host G. emersonii and endoparasite A. protococcarum is illustrated in Figure 6.

\subsection{Defense Strategies in G. emersonii}

Plant defense against pathogenic fungi is based on a combination of the innate immune system, which includes a basal defense system based on pattern-recognition receptors (PRRs) that elicit PAMP-triggered immunity (PTI) and a more specialized recognition system based on resistance proteins (R proteins) that activate effector-triggered immunity (ETI) $[16,17]$. First, plant host immunity uses PRR, such as leucine-rich repeat kinases (LRR) and lysine motif (LysM) kinase receptors, to identify pathogens that attack the host. Transcriptome analysis of the red alga P. yezoensis during oomycete infection revealed that three lectin genes (PRRs) were upregulated after infection, whereas EGF- and LysMcontaining genes could not be found [14]. The results show that microalga G. emersonii 
transcripts contained potential cellular receptors, including EGF, LRR, and LysM genes and that the expression of six LRR-containing genes was significantly upregulated in the infected G. emersonii cells. Second, plant resistance (R) proteins recognize the effectors of pathogens, activate signaling, and trigger ETI [18]. Recent studies have identified six genes encoding R proteins, including the nucleotide binding site (NBS) domain, that were identified in the genome of Chromochloris zofingiensis, and the fusion events of the NBS and LRR domains might occur in Chlorophyta and plants [19]. In G. emersonii, four genes encoding putative $\mathrm{R}$ proteins with an LRR domain showed no significant change during infection, indicating that endoparasite $A$. protococcarum can somehow avoid or inhibit this dense system. This might support the idea that ETI avoidance can be a crucial virulence strategy for plant pathogens [17]. Moreover, previous studies have confirmed the roles of large HSPs in recognizing plant pathogen effectors and activating defense responses against pathogens $[13,14]$. For instance, HSP70 and HSP90 might serve as R proteins that control plant disease resistance [20]. In our study, the genes encoding HSP70 and HSP90 were upregulated in G. emersonii during infection. Moreover, the genes encoding other key enzymes, such as HSP, which are involved in the endocytosis pathway, were induced. These results suggest that the upregulated genes encoding HSP70 and HSP90 might play a vital role in G. emersonii defense responses.

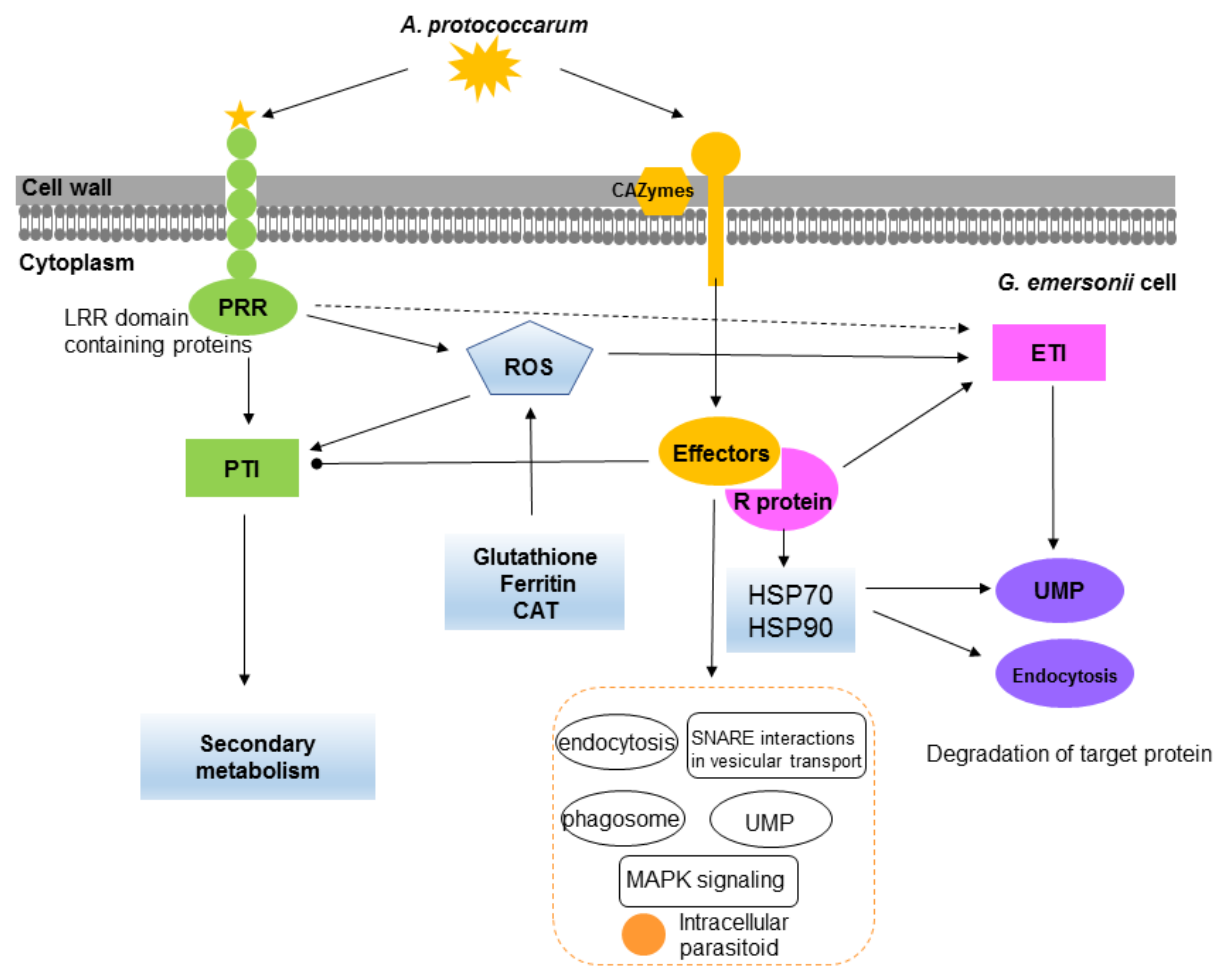

Figure 6. Schematic diagram showing the basic interaction of microalga G. emersonii and endoparasite A. protococcarum. Similar to plant, G. emersonii cells can perceive pathogen via potential PRRs and initiate PTI. Reactive oxygen species (ROS) was rapidly induced in response to infection. Meanwhile, ROS scavenging-related genes (glutathione, ferritin, CAT) were upregulated to eliminate excess ROS. Several HSP70 and HSP90 were upregulated and may serve as putative R genes to recognize the effectors of pathogens and trigger ETI. The ubiquitin-proteasome system (UPS) and endocytosis, which may be involved in regulating apoptotic cell death, were also upregulated to response to the infection. For endoparasite A. protococcarum, genes for carbohydrate-active enzymes, pathogenhost interaction, and putative effectors are significantly upregulated during infection. Endocytosis, phagosome, ubiquitin-mediated proteolysis, and SNARE interactions in vesicular transport pathway represent the KEGG enrichment pathways involved in the modulation of vacuole transport of intracellular parasitoid. 
The generation of ROS plays an important role in the PTI and ETI of plants as signaling molecules [21]. Recent study has demonstrated that PRR and co-receptors are required for ROS generation, which is a key signaling event combining PRR- and NLR-mediated immunity [22]. In this study, the ROS level gradually increased during infection, suggesting that $G$. emersonii has a signaling system that induces ROS in these cells when infected with fungal parasites. Based on STEM analysis, the genes in cluster VI were enriched with many oxidoreductase-related terms, which were upregulated or stable in the ES and MS stages and then downregulated in the LS stage. At the same time, plants might induce enzymes in response to excess ROS to protect their cells. It has been reported that microalgae can induce a variety of ROS scavenging enzymes due to oxidative stress [23]. Our data suggest that the genes associated with ROS-scavenging enzymes were induced in ES, MS, and LS. Notably, the expression of the genes encoding glutathione, ferritin, and CAT was upregulated dramatically, suggesting that the upregulation of ROS scavengingrelated genes may be involved in defensive responses. These results denote that ROS production alone is insufficient to stop the attack of endoparasite $A$. protococcarum, as the ROS generation might also be affected by ROS scavenging-related genes.

In plants, the ubiquitin-proteasome system (UPS) plays a crucial role in the control of plant immune signaling against pathogens [24]. In the UPS, damaged or superfluous proteins are mediated by three types of ubiquitin enzymes: E1 (ubiquitin-activating enzyme), E2 (ubiquitin-conjugating enzyme), and E3 (ubiquitin-protein ligase) [25]. Previous studies have concluded that ubiquitin-mediated proteolysis may be involved in regulating apoptotic cell death [26]. Our results show that cluster I was enriched in the ubiquitinmediated proteolysis pathway. In G. emersonii, the expression of genes encoding enzymes E1 and E2 was significantly increased, especially in MS. In contrast, the genes related to E3 were found to be downregulated. These results hinted that UPS might be involved in the G. emersonii response to endoparasite A. protococcarum. Furthermore, recent findings have suggested that pathogens may manipulate the ubiquitin pathway of host cells to accelerate infection using effectors [27].

The expression of downstream TFs was also regulated. Several studies have reported that TF families, including WRKY, MYB, ERF, and bZIP, can play vital roles in signal transduction and plant defense [28,29]. In this study, SET, SNF2, GNAT, MYB, AP2-EREBP, and TRAF were the top six differentially expressed TFs in G. emersonii. However, only five TFs were upregulated during infection: two SNF2, one PLATZ, one FHA, and one orphan (Table 1). The TFs were differentially expressed in G. emersonii cells during infection, implying that these TFs might be required in the establishment of G. emersonii defense to endoparasite $A$. protococcarum.

Together, the upregulation of these genes associated with response regulation suggests that G. emersonii has a conserved defense system against endoparasite A. protococcarum.

\subsection{Pathogenicity of A. protococcarum}

For pathogenic fungi invading a host, carbohydrate-active enzymes (CAZymes) play important roles in penetration, colonization, exit, and dispersal during infection progress [30]. Several studies have indicated that massive CAZyme-encoding genes are activated during infection [31,32]. For endoparasite A. protococcarum, they need a living microalgal host to complete their life cycle (Ding et al., 2017). In other words, they need to use CAZymes to degrade the cell walls of G. emersonii to colonize. In this study, many CAZymeencoding genes were differentially expressed and identified in cluster II of A. protococcarum. Notably, two genes encoding $\alpha$-glucosidase were upregulated by 6.2- and 10.7-log2 fold changes in LS, respectively. Many genes encoding trehalase, cellulase, mannan, and several chitin-related genes also showed significant increases. Comprehensive GO enrichment and STEM results suggest that the genes in cluster III were enriched in the establishment of localization in cell and protein localization. In the LS, endoparasite A. protococcarum was widely distributed in the microalgal host, thus the expression of genes involved in the 
establishment of localization were decreased. This group of genes might play a positive role in the pathogenicity of $A$. protococcarum.

Pathogens have unique virulence mechanisms that target and manipulate the host immune response to promote pathogenesis [33]. It is acknowledged that fungal pathogens can produce effectors to suppress PTI and ETI signaling and promote pathogen virulence [16], thus leading to successful proliferation. A recent study has suggested that pathogens can maximize pathogenicity benefits and avoid host detection through regulating effector dosage [17]. In A. protococcarum, 72 genes associated with increased virulence and 15 genes associated with effectors were upregulated in all the infection stages. Among them, 11 putative secretory protein-encoding genes were identified in the pathogen-host interaction genes. A candidate hypervirulence gene (Cluster-8366.11022) and two candidate effectors (Cluster-9881.0, Cluster-8366.6798) were upregulated during infection. These results suggest that these genes are involved in the pathogenicity of $A$. protococcarum.

The comprehensive KEGG enrichment and STEM results suggest that the genes in cluster II, which showed an upregulated temporal trend, were closely related to endocytosis, phagosome, ubiquitin-mediated proteolysis, and SNARE interactions in vesicular transport pathways. These pathways may be involved in the modulation of vacuole transport, which is crucial for endoparasite survival and replication within the host [34]. Moreover, the mitogen-activated protein kinase (MAPK) signaling pathway was significantly enriched in cluster II. The MAPK signaling pathway is involved in the pathogenicity of plant pathogenic fungi [35]. Based on GO enrichment, genes related to the regulation of GTPase activity and response to host immunity, those genes that showed an upregulated temporal trend, were in cluster II. Given all the results together, we argue that endoparasite A. protococcarum possessed a robust pathogenicity, which can inhibit the expression of defense responses in G. emersonii to successfully infect the host.

\section{Materials and Methods}

\subsection{Microalga and Parasite Laboratory Culturing}

The alga strain G. emersonii was acquired from the Algae Culture Collection at the Wuhan Botanical Garden, Chinese Academy of Sciences, China. G. emersonii cells were cultivated in modified BG11 medium, under laboratory conditions, at $26 \pm 1{ }^{\circ} \mathrm{C}$ under $70 \mu \mathrm{mol}$ photons $\mathrm{m}^{-2} \mathrm{~s}^{-1}$ illumination for $14 \mathrm{~h}$ every day.

The algal parasite A. protococcarum WZ01 was isolated from an open raceway pond of G. emersonii culture in Chenghai, China [11]. A. protococcarum WZ01 is an endoparasite and must be fed with G. emersonii. During the experiment, A. protococcarum WZ01 was maintained as a highly infective parasite through the addition of WZ01-infected G. emersonii filtrate $(50 \mu \mathrm{L})$ to healthy G. emersonii culture $(20 \mathrm{~mL})$ every seven days. The dual-cultures were cultivated at $28^{\circ} \mathrm{C}$ under illumination of $20 \mu \mathrm{mol}$ photons $\mathrm{m}^{-2} \mathrm{~s}^{-1}$ with a $14 \mathrm{~h}: 10 \mathrm{~h}$ light/dark cycle.

\subsection{Infection Test and Sampling}

G. emersonii cells were cultivated in $600 \mathrm{~mL}$ of modified BG11 medium in a photobioreactor with aeration of $1 \%(v / v) \mathrm{CO}_{2}$ at $28^{\circ} \mathrm{C}$ under illumination of $140 \mu \mathrm{mol}$ photons $\mathrm{m}^{-2} \mathrm{~s}^{-1}$ with a $14 \mathrm{~h}: 10 \mathrm{~h}$ light/dark cycle. Cells in the exponential growth phase were harvested and used for the infection experiments. The initial cell densities of G. emersonii in the tubular photobioreactors were $\mathrm{OD}_{540}=0.10 \pm 0.01$. Then, $0.05 \%$ volume of the axenic $A$. protococcarum WZ01 filtrate through a $5 \mu \mathrm{m}$ membrane filter (Whatman, Maidstone, UK) was added to the G. emersonii culture. Mixed cell cultures were cultivated under the same conditions and used as controls. The infected samples and controls had three replicates.

After inoculation, the infection rate was determined using an Olympus BX51 microscope with hemocytometers. The infection rate was quantified as described by Ding et al. [4]. Samples were collected at 1, 2, 3, 4, and 5 days post-inoculation (dpi) for further analysis. 


\subsection{Chlorophyll Fluorescence Parameter and ROS Measurement}

During the experiments, the maximum Fv/Fm (maximum photochemical yield) was measured using a PAM 2500 fluorometer, as described in [36]. ROS levels in G. emersonii cells were detected using an ROS assay kit (S0033S, Beyotime, China). Briefly, $5 \mathrm{~mL}$ of mixed cell culture was centrifuged at $5000 \times \mathrm{g}$ for $5 \mathrm{~min}$, and the cell pellets were resuspended in $1 \mathrm{~mL}$ basal medium containing $10 \mu \mathrm{M}$ DCFH-DA. The suspension was incubated in the dark at $37^{\circ} \mathrm{C}$ for $30 \mathrm{~min}$. After labeling, the cells were washed three times with basal medium and resuspended in the original culture medium. Subsequently, the cell suspension was transferred to each well of a black microtiter plate (Greiner Bio, Chimney well, Germany). Fluorescence was analyzed using a fluorescence microplate reader (TECAN, M200 PRO) with excitation and emission wavelengths of 488 and $525 \mathrm{~nm}$, respectively. For each treatment and control, three replicates were performed.

\subsection{RNA Isolation, cDNA Library Construction, and Sequencing}

The control or infected samples $(200 \mathrm{~mL})$ were harvested by centrifuging at $5000 \times \mathrm{g}$ for $5 \mathrm{~min}$. The cell pellets were frozen in liquid nitrogen and maintained at $-80^{\circ} \mathrm{C}$ until RNA extraction. Three replicates were prepared for each treatment and control. Total RNA from each sample was isolated using TRIzol reagent (Invitrogen, Carlsbad, CA, USA) according to the manufacturer's instructions. Total RNA samples were quantified using a Nanodrop 2000 instrument (Thermo Scientific, San Jose, DE, USA) and the RNA Nano 6000 Assay Kit using the Agilent Bioanalyzer 2100 system (Agilent Technologies, Santa Clara, CA, USA) and Qubit RNA Assay Kit in Qubit 2.0 Fluorometer (Life Technologies, Carlsbad, CA, USA) and 1\% agarose gels. A total of $1.5 \mu \mathrm{g}$ RNA from each sample was used for cDNA library construction and RNA sequencing on an Illumina Novaseq 6000 platform with a $150 \mathrm{bp}$ pair-ended strategy, which was performed at the Novogene Bioinformatics Institute (Beijing, China).

\subsection{Transcriptome Data Analysis}

Raw reads were processed before downstream processing to derive clean reads by removing adaptor sequences, empty reads, and low-quality reads $(<\mathrm{Q} 20)$. For the host, the clean reads were mapped to the G. emersonii reference genome using HISAT. For the pathogen, de novo transcriptome assembly was performed using Trinity [37] because of the lack of genomic information, and gene function annotation was performed in $\mathrm{Nr}, \mathrm{Nt}$, Pfam, KOG/COG, Swiss-Prot, KEGG, and GO databases. The host algae and endoparasite transcriptomes were subjected to differential expression analysis by calculating the target FPKM [38], thus allowing comparisons between different infection stages. Differentially expressed genes (DEGs) were identified using the DESeq method based on the Benjamini and Hochberg approach. The DEGs were deemed with a padj $<0.05$ and $\mid \log _{2}$ (fold change) $\mid>1$ in sequence counts between the different comparison groups. Venn diagrams of host and fungal parasites were used to elucidate the differential gene expression patterns between different infection stages.

Short Time-series Expression Miner (STEM) software was used for clustering analysis based on the similar expression trend patterns in G. emersonii and A. protococcarum. Novomagic, a free online platform, was used for GO enrichment, KEGG pathway analysis, and hierarchical clustering heatmap analysis (https:/ / magic.novogene.com, Access date: 13 July 2021). The significantly enriched GO terms and KEGG pathways were determined with a $p$-value of $<0.05$. To identify genes encoding CAZymes of $A$. protococcarum involved in the infection progress, we applied the dbCAN2 meta server ( http:/ /bcb.unl.edu/dbCAN2/blast.php, Access date: 22 July 2021) and Carbohydrate Active Enzymes database (http:/ / www.cazy.org, Access date: 22 July 2021) with a BLAST e-value cutoff of $1 \times 10^{-5}$ for analysis. Pathogen-host interaction genes were annotated using PHI-base [39]. PFAM annotation of each gene was performed using the Pfam database (http://pfam.xfam.org/, Access date: 23 July 2021). To predict secretory proteins, the 
SignalP 4.0 (Petersen et al. 2011), TMHMM v. 2.0, TargetP-2.0 Server, and kohgpi-1.5 programs (GPI-SOM database) were used.

\section{Conclusions}

In summary, this is the first report on microalgae-endoparasite interaction at the transcriptional level. For microalgal hosts, STEM and functional analysis indicated that $A$. protococcarum infection triggers dynamic defense responses in the microalga G. emersonii. Potential pattern recognition receptors, ROS-scavenging enzymes, large HSPs, TFs, and the ubiquitin-proteasome system of G. emersonii all may be required for defense against endoparasite A. protococcarum. For endoparasite, transcriptome analysis revealed that the expression of genes associated with pathogenicity were upregulated significantly, such as CAZyme-encoding genes, PHI-base genes, and putative effectors. These results lay a foundation for understanding microalgal host defense strategies and parasite pathogenicity, which is crucial for preventing and controlling microalgal diseases.

Supplementary Materials: The following are available online at https:/ /www.mdpi.com/article/10 $.3390 / \mathrm{ijms} 222312847 / \mathrm{s} 1$.

Author Contributions: Conceptualization, Y.D.; methodology, Y.D.; software, Y.D.; formal analysis, Y.D. and Z.W.; investigation, Y.D. and Y.W.; resources, Y.G.; writing—original draft preparation, Y.D.; writing—review and editing, Y.D. and X.W.; supervision, Y.L.; funding acquisition, Y.D. All authors have read and agreed to the published version of the manuscript.

Funding: This research was funded by the National Natural Science Foundation of China, grant number 32072998; the Natural Science Foundation of Hubei Province, grant number 2020CFB733; and the National Natural Science Foundation of China, grant number 32172999.

Institutional Review Board Statement: Not applicable.

Informed Consent Statement: Not applicable.

Data Availability Statement: The RNA sequencing read data were deposited in the GenBank SRA database under the accession number PRJNA753980.

Acknowledgments: We would like to thank the Novogene Bioinformatics Institute (Beijing, China) for technical assistance with bioinformatics and Novomagic platform usage.

Conflicts of Interest: The authors declare no conflict of interest.

\section{List of Abbreviations}

CAZymes carbohydrate-active enzymes

DEGs differentially expressed genes

EGF epidermal growth factor

ETI effector-triggered immunity

ES early stage

$\mathrm{Fv} / \mathrm{Fm}$ maximum photochemical yield

GA control

HSPs large heat shock proteins

LRR leucine-rich repeat

LysM lysine motif

LS late stage

MS medium stage

MAPK mitogen-activated protein kinase

NBS nucleotide binding site

PTI PAMP-triggered immunity

PRRs pattern recognition receptors

$R$ proteins resistance proteins

ROS reactive oxygen species

STEM short time-series expression miner

UPS ubiquitin-proteasome system 


\section{References}

1. Chen, J.; Li, J.; Dong, W.; Zhang, X.; Tyagi, R.D.; Drogui, P.; Surampalli, R.Y. The potential of microalgae in biodiesel production. Renew. Sustain. Energy Rev. 2018, 90, 336-346. [CrossRef]

2. Kondaveeti, S.; Abu-Reesh, I.M.; Mohanakrishna, G.; Bulut, M.; Pant, D. Advanced routes of biological and bio-electrocatalytic carbon dioxide $\left(\mathrm{CO}_{2}\right)$ mitigation toward carbon neutrality. Front. Energy Res. 2020, 8, 94. [CrossRef]

3. Kumsiri, B.; Pekkoh, J.; Pathom-aree, W.; Lumyong, S.; Phinyo, K.; Pumas, C.; Srinuanpan, S. Enhanced production of microalgal biomass and lipid as an environmentally friendly biodiesel feedstock through actinomycete co-culture in biogas digestate effluent. Bioresour. Technol. 2021, 337, 125446. [CrossRef]

4. Ding, Y.; Wen, X.; Peng, X.; Zhang, A.; Wang, Z.; Geng, Y.; Li, Y. Surfactants as fungal parasite control agents in oleaginous microalga, Graesiella sp. WBG-1, mass culture. Algal Res. 2019, 41, 101539. [CrossRef]

5. Sime-Ngando, T. Phytoplankton chytridiomycosis: Fungal parasites of phytoplankton and their imprints on the food web dynamics. Front. Microbiol. 2012, 3, 361. [CrossRef]

6. Ding, Y.; Peng, X.; Wang, Z.; Wen, X.; Geng, Y.; Zhang, D.; Li, Y. Occurrence and characterization of an epibiotic parasite in cultures of oleaginous microalga Graesiella sp. WBG-1. J. Appl. Phycol. 2018, 30, 819-830. [CrossRef]

7. Letcher, P.M.; Lopez, S.; Schmieder, R.; Lee, P.A.; Behnke, C.; Powell, M.J.; Mcbride, R.C. Characterization of Amoeboaphelidium protococcarum, an algal parasite new to the cryptomycota isolated from an outdoor algal pond used for the production of biofuel. PLoS ONE 2013, 8, e56232. [CrossRef]

8. Frenken, T.; Alacid, E.; Berger, S.A.; Bourne, E.C.; Gerphagnon, M.; Grossart, H.P.; Gsell, A.S.; Ibelings, B.W.; Kagami, M.; Kupper, F.C.; et al. Integrating chytrid fungal parasites into plankton ecology: Research gaps and needs. Environ. Microbiol. 2017, 9, 3802-3822. [CrossRef] [PubMed]

9. Lin, J.; Yan, H.; Zhao, L.; Li, Y.; Nahidian, B.; Zhu, M.; Hu, Q.; Han, D. Interaction between the cell walls of microalgal host and fungal carbohydrate-activate enzymes is essential for the pathogenic parasitism process. Environ. Microbiol. 2021, 23, 5114-5130. [CrossRef]

10. Wen, X.; Tao, H.; Peng, X.; Wang, Z.; Ding, Y.; Xu, Y.; Liang, L.; Du, K.; Zhang, A.; Liu, C.; et al. Sequential phototrophicmixotrophic cultivation of oleaginous microalga Graesiella sp. WBG-1 in a $1000 \mathrm{~m} 2$ open raceway pond. Biotechnol. Biofuels 2019, 12, 27. [CrossRef] [PubMed]

11. Ding, Y.; Peng, X.; Wang, Z.; Wen, X.; Geng, Y.; Li, Y. Isolation and characterization of an endoparasite from the culture of oleaginous microalga Graesiella sp. WBG-1. Algal Res. 2017, 26, 371-379. [CrossRef]

12. Strittmatter, M.; Grenville-Briggs, L.J.; Breithut, L.; Van West, P.; Gachon, C.M.M.; Küpper, F.C. Infection of the brown alga Ectocarpus siliculosus by the oomycete Eurychasma dicksoniiinduces oxidative stress and halogen metabolism. Plant Cell Environ. 2016, 39, 259-271. [CrossRef]

13. Im, S.H.; Klochkova, T.A.; Lee, D.J.; Gachon, C.M.M.; Kim, G.H. Genetic toolkits of the red alga Pyropia tenera against the three most common diseases in Pyropia farms. J. Phycol. 2019, 55, 801-815. [CrossRef]

14. Tang, L.; Qiu, L.P.; Liu, C.; Du, G.Y.; Mo, Z.L.; Tang, X.H.; Mao, Y.X. Transcriptomic insights into innate immunity responding to red rot disease in red alga Pyropia yezoensis. Int. J. Mol. Sci. 2019, 20, 5970. [CrossRef] [PubMed]

15. Smith, V.H.; McBride, R.C.; Shurin, J.B.; Bever, J.D.; Crews, T.E.; Tilman, G.D. Crop diversification can contribute to disease risk control in sustainable biofuels production. Front. Ecol. Environ. 2015, 13, 561-567. [CrossRef]

16. Dangl, J.L.; Horvath, D.M.; Staskawicz, B.J. Pivoting the plant immune system from dissection to deployment. Science 2013, 341, 746-751. [CrossRef] [PubMed]

17. Laflamme, B.; Dillon, M.M.; Martel, A.; Almeida, R.N.D.; Desveaux, D.; Guttman, D.S. The pan-genome effector-triggered immunity landscape of a host-pathogen interaction. Science 2020, 367, 763-768. [CrossRef]

18. Presti, L.L.; Lanver, D.; Schweizer, G.; Tanaka, S.; Liang, L.; Tollot, M.; Zuccaro, A.; Reissmann, S.; Kahmann, R. Fungal Effectors and Plant Susceptibility. Annu. Rev. Plant Biol. 2015, 66, 513-545. [CrossRef]

19. Shao, Z.Q.; Xue, J.Y.; Wang, Q.; Wang, B.; Chen, J.Q. Revisiting the origin of plant NBS-LRR genes. Trends Plant Sci. 2019, $24,9-12$. [CrossRef]

20. Thao, N.P.; Chen, L.; Nakashima, A.; Hara, S.I.; Umemura, K.; Takahashi, A.; Shirasu, K.; Kawasaki, T.; Shimamoto, K. RAR1 and HSP90 form a complex with Rac/Rop GTPase and function in innate-immune responses in rice. Plant Cell. 2007, 19, 4035-4045. [CrossRef]

21. Yoshioka, H.; Adachi, H.; Nakano, T.; Miyagawa, N.; Asai, S.; Ishihama, N.; Yoshioka, M. Hierarchical regulation of NADPH oxidase by protein kinases in plant immunity. Physiol. Mol. Plant Pathol. 2016, 95, 20-26. [CrossRef]

22. Yuan, M.; Jiang, Z.; Bi, G.; Nomura, K.; Liu, M.; Wang, Y.; Cai, B.; Zhou, J.M.; He, S.Y.; Xin, X.F. Pattern-recognition receptors are required for NLR-mediated plant immunity. Nature 2021, 592, 105-109. [CrossRef]

23. Hu, C.; Cui, D.; Sun, X.; Shi, J.; Song, L.; Li, Y.; Xu, N. Transcriptomic analysis unveils survival strategies of autotrophic Haematococcus pluvialis against high light stress. Aquaculture 2019, 513, 734430. [CrossRef]

24. Dubiella, U.; Serrano, I. The Ubiquitin proteasome system as a double agent in plant-virus interactions. Plants 2021, 10, 928. [CrossRef] [PubMed]

25. Su, T.; Yang, M.Y.; Wang, P.P.; Zhao, Y.X.; Ma, C.L. Interplay between the ubiquitin proteasome system and ubiquitin-mediated autophagy in plants. Cells 2020, 9, 2219. [CrossRef] 
26. Gupta, I.; Singh, K.; Varshney, N.K.; Khan, S. Delineating crosstalk mechanisms of the ubiquitin proteasome system that regulate apoptosis. Front. Cell Dev. Biol. 2018, 6, 11. [CrossRef]

27. Kud, J.; Wang, W.J.; Gross, R.; Fan, Y.H.; Huang, L.; Yuan, Y.L.; Gray, A.; Duarte, A.; Kuhl, J.C.; Caplan, A.; et al. The potato cyst nematode effector RHA1B is a ubiquitin ligase and uses two distinct mechanisms to suppress plant immune signaling. PLoS Pathog. 2019, 15, e1007720. [CrossRef] [PubMed]

28. Wang, Y.; Huang, L.; Luo, W.; Jin, Y.; Gong, F.; He, J.; Liu, D.; Zheng, Y.; Wu, B. Transcriptome analysis provides insights into the mechanisms underlying wheat cultivar Shumai 126 responding to stripe rust. Gene 2021, 768, 145290. [CrossRef]

29. Du, F.; Hu, C.; Sun, X.; Xu, N. Transcriptome analysis reveals pathways responsible for the promoting effect of sucrose on astaxanthin accumulation in Haematococcus pluvialis under high light condition. Aquaculture 2021, 530, 735757. [CrossRef]

30. De Vries, S.; de Vries, J. A global survey of carbohydrate esterase families 1 and 10 in oomycetes. Front. Genet. 2020, 11, 756. [CrossRef] [PubMed]

31. Sophie de Vries, J.d.V.; Archibald, J.M.; Slamovits, C.H. Comparative analyses of saprotrophy in Salisapilia sapeloensis and diverse plant pathogenic oomycetes reveal lifestyle-specific gene expression. FEMS Microbiol. Ecol. 2020, 96, fiaa184. [CrossRef]

32. Grams, N.; Komar, H.; Jainchill, D.; Ospina-Giraldo, M. Comparative expression analysis of Phytophthora sojae polysaccharide lyase family 3 (pectate lyase) genes during infection of the soybean Glycine max. Phytopathol. Res. 2019, 1, 15. [CrossRef]

33. Baddal, B. Next-generation technologies for studying host-pathogen interactions: A focus on dual transcriptomics, CRISPR/Cas9 screening and organs-on-chips. Pathog. Dis. 2019, 77, ftz060. [CrossRef]

34. Arasaki, K.; Roy, C.R. Legionella pneumophila promotes functional interactions between plasma membrane syntaxins and sec22b. Traffic 2010, 11, 587-600. [CrossRef]

35. Cheng, B.; Yu, X.; Ma, Z.; Dong, S.; Dou, D.; Wang, Y.; Zheng, X. Phytophthora sojae effector Avh331 suppresses the plant defence response by disturbing the MAPK signalling pathway. Physiol. Mol. Plant Pathol. 2012, 77, 1-9. [CrossRef]

36. Wen, X.; Zhang, A.; Zhu, X.; Liang, L.; Huo, Y.; Wang, K.; Yu, Y.; Geng, Y.; Ding, Y.; Li, Y. Controlling of two destructive zooplanktonic predators in Chlorella mass culture with surfactants. Biotechnol. Biofuels 2021, 14, 21. [CrossRef] [PubMed]

37. Grabherr, M.G.; Haas, B.J.; Yassour, M.; Levin, J.Z.; Thompson, D.A.; Amit, I.; Adiconis, X.; Fan, L.; Raychowdhury, R.; Zeng, Q.; et al. Full-length transcriptome assembly from RNA-Seq data without a reference genome. Nat. Biotechnol. 2011, 29, 644-652. [CrossRef]

38. Trapnell, C.; Roberts, A.; Goff, L.; Pertea, G.; Kim, D.; Kelley, D.R.; Pimentel, H.; Salzberg, S.L.; Rinn, J.L.; Pachter, L. Differential gene and transcript expression analysis of RNA-seq experiments with TopHat and Cufflinks. Nat. Protoc. 2012, 7, 562-578. [CrossRef] [PubMed]

39. Urban, M.; Cuzick, A.; Seager, J.; Wood, V.; Rutherford, K.; Venkatesh, S.Y.; De Silva, N.; Martinez, M.C.; Pedro, H.; Yates, A.D.; et al. PHI-base: The pathogen-host interactions database. Nucleic Acids Res. 2020, 48, D613-D620. [CrossRef] 